ACCEPTED BY ApJ Letters, 2017 June 01

Preprint typeset using $\mathrm{LAT}_{\mathrm{E}} \mathrm{X}$ style AASTeX6 v. 1.0

\title{
THE YOUNG L DWARF 2MASS J11193254-1137466 IS A PLANETARY-MASS BINARY
}

\author{
William M. J. Best ${ }^{1}$, Michael C. Liu ${ }^{1}$, Trent J. Dupuy ${ }^{2}$, Eugene A. Magnier ${ }^{1}$ \\ ${ }^{1}$ Institute for Astronomy, University of Hawaii, 2680 Woodlawn Drive, Honolulu, HI 96822, USA; wbest@ifa.hawaii.edu \\ ${ }^{2}$ University of Texas at Austin, Department of Astronomy, 2515 Speedway C1400, Austin, TX 78712, USA
}

\section{ABSTRACT}

We have discovered that the extremely red, low-gravity L7 dwarf 2MASS J11193254-1137466 is a $0.14^{\prime \prime}$ (3.6 AU) binary using Keck laser guide star adaptive optics imaging. 2MASS J11193254-1137466 has previously been identified as a likely member of the TW Hydrae Association (TWA). Using our updated photometric distance and proper motion, a kinematic analysis based on the BANYAN II model gives an $82 \%$ probability of TWA membership. At TWA's $10 \pm 3 \mathrm{Myr}$ age and using hot-start evolutionary models, 2MASS J11193254-1137466AB is a pair of $3.7_{-0.9}^{+1.2} M_{\text {Jup }}$ brown dwarfs, making it the lowest-mass binary discovered to date. We estimate an orbital period of $90_{-50}^{+80}$ years. One component is marginally brighter in $K$ band but fainter in $J$ band, making this a probable flux-reversal binary, the first discovered with such a young age. We also imaged the spectrally similar TWA L7 dwarf WISEA J114724.10-204021.3 with Keck and found no sign of binarity. Our evolutionary model-derived $T_{\text {eff }}$ estimate for WISEA J114724.10-204021.3 is $\approx 230 \mathrm{~K}$ higher than for 2MASS J11193254-1137466AB, at odds with their spectral similarity. This discrepancy suggests that WISEA J114724.10-204021.3 may actually be a tight binary with masses and temperatures very similar to 2MASS J11193254-1137466AB, or further supporting the idea that near-infrared spectra of young ultracool dwarfs are shaped by factors other than temperature and gravity. 2MASS J11193254-1137466AB will be an essential benchmark for testing evolutionary and atmospheric models in the young planetary-mass regime.

Keywords: brown dwarfs - binaries: close — stars: individual (2MASS J11193254-1137466, WISEA J114724.10-204021.3)

\section{INTRODUCTION}

Brown dwarfs with masses $\lesssim 15 M_{\text {Jup }}$ and ages $\lesssim 100$ Myr lie at a nexus of astronomical interest. They represent the lowest-mass and youngest products of star formation, and as such offer rare empirical tests for evolutionary and atmospheric models. They are also the best field analogs to directly-imaged giant exoplanets, which are far more difficult to directly observe due to the glare of their host stars.

Brown dwarfs cool continuously as they age, and the resulting mass-age-luminosity degeneracy makes their physical properties challenging to infer without constraints on at least two of those three parameters. The atmospheres of young brown dwarfs exhibit clear spectral signatures of low gravity (Cruz et al. 2009; Allers \& Liu 2013), but the age calibration for these signatures lacks precision better than $\approx 100 \mathrm{Myr}$ (e.g., Liu et al. 2016). Some of the lowest-mass objects have been identified as members of nearby young moving groups (e.g., Gagné et al. 2014), which provide much tighter age constraints and thus more precise mass es- timates from evolutionary models than for ordinary field objects. Young binaries with small separations are even more useful as benchmarks, as their orbits can yield model-independent dynamical masses, providing exacting tests for models (e.g., Dupuy et al. 2009; Dupuy \& Liu 2017).

2MASS J11193254-1137466 (a.k.a. TWA 42; hereinafter 2MASS J1119-1137) was discovered by Kellogg et al. (2015, hereinafter K15) in a search for $\mathrm{L}$ and $\mathrm{T}$ dwarfs with unusual photometry. 2MASS J1119-1137 is an L7 dwarf with extremely red optical and near-IR colors along with spectral signatures of low gravity indicating youth (K15; Kellogg et al. 2016, hereinafter K16). K16 identified 2MASS J1119-1137 as a candidate member of the TW Hydrae Association (TWA; Webb et al. 1999), whose age implies a mass of only 4.3-7.6 $M_{\text {Jup }}$ for this object. 2MASS J1119-1137 would be one of the two lowest-mass isolated members of TWA, comparable only to the L7 dwarf WISEA J114724.10-204021.3 (hereinafter WISEA J1147-2040; $6.6 \pm 1.9 \quad M_{\text {Jup }}$; Schneider et al. 2016; Faherty et al. 2016), and among 
the lowest-mass free-floating brown dwarfs known.

We are conducting a high angular-resolution imaging survey of nearby brown dwarfs to identify binaries. In this Letter we show that 2MASS J1119-1137 is a nearly equal-flux binary with component masses in the planetary regime.

\section{OBSERVATIONS}

We observed 2MASS J1119-1137 on 2016 November 25 UT using the laser guide star adaptive optics (LGS AO) system at the Keck II Telescope (van Dam et al. 2006; Wizinowich et al. 2006). We used the facility infrared camera NIRC2 in its narrow fieldof-view configuration, using the $R=13.5 \mathrm{mag}$ field star USNO-B1.0 0783-0249513 (Monet et al. 2003) located $35^{\prime \prime}$ from 2MASS J1119-1137 for tip-tilt correction. Skies were mostly clear, with $K$-band seeing of $1.6^{\prime \prime}$ measured contemporaneously at UKIRT. We obtained 3 dithered images at $K$ band in which 2MASS J1119-1137 appeared to be an equal-flux binary. We observed 2MASS J1119-1137 again on 2017 March $18 \mathrm{UT}$ in $Y J H K$ bands using the same configuration, under clear skies with seeing $\approx 0.5^{\prime \prime}$ as measured by the differential image motion monitor (DIMM) at the Canada-France-Hawaii Telescope (CFHT). Details are in Table 1.

In addition, we observed WISEA J1147-2040 on 2016 May 18 UT with a similar configuration using the $R=17.4 \mathrm{mag}$ field star USNO-B1.0 0693-0264226 (Monet et al. 2003) located $66^{\prime \prime}$ away for tip-tilt correction, under clear skies with seeing $\approx 0.5^{\prime \prime}$ from DIMM. The target appeared to be a single object at $0.11^{\prime \prime}$ resolution.

We reduced and analyzed our data using the methods described in, e.g., Liu et al. (2008) and Dupuy \& Liu (2017). Briefly, we calibrated our images using flat fields and dark frames, performed sky subtraction, and registered and stacked images to form a final mosaic for each epoch and filter (Figure 1). We measured the relative astrometry and flux ratios of the binary by fitting a PSF model to the individual images, applying the NIRC2 pixel scale, orientation, and distortion correction from Service et al. (2016). For images in which the components were well separated (all but $Y$ band), we used the StarFinder software package (Diolaiti et al. 2000) to simultaneously solve for an empirical PSF and binary parameters. For $Y$ band, we used an analytical PSF of two elliptical three-component Gaussians. We applied additional corrections for differential aberration and atmospheric refraction. We used the rms of the measurements from individual images as the uncertainties on the separation, position angle (PA), and $\Delta \mathrm{mag}$ of the binary components (Table 1), adding the errors in plate scale $(0.4 \%)$ and orientation (0.020 deg) from Service et al. (2016) in quadrature.

Table 1. Keck LGS AO Observations

\begin{tabular}{|c|c|c|c|c|c|c|c|c|c|c|c|c|}
\hline & Object & & $\begin{array}{l}\text { Date } \\
\text { (UT) }\end{array}$ & Filter & $N$ & $\begin{array}{l}t_{\text {int }} \\
(\mathrm{sec})\end{array}$ & Airmass & $\begin{array}{c}\text { FWHM }^{\mathrm{a}} \\
(\mathrm{mas})\end{array}$ & Strehl ratio ${ }^{a}$ & $\begin{array}{l}\text { Separation } \\
\text { (mas) }\end{array}$ & $\begin{array}{c}\text { Position angle } \\
\text { (deg) }\end{array}$ & $\Delta \mathrm{mag}$ \\
\hline \multirow[t]{5}{*}{ 2MASS } & J11193254-1137466 & 2016 & 6 Nov 25 & $K_{\mathrm{MKO}}$ & 3 & 60 & 1.26 & $94 \pm 3$ & $0.148 \pm 0.015$ & $137.8 \pm 1.7$ & $239.2 \pm 0.5$ & $0.125 \pm 0.010$ \\
\hline & & 2017 & 7 Mar 18 & $K_{\mathrm{MKO}}$ & 6 & 60 & 1.17 & $59 \pm 2$ & $0.41 \pm 0.04$ & $138.10 \pm 0.10$ & $239.08 \pm 0.07$ & $0.027 \pm 0.010$ \\
\hline & & & & $H_{\mathrm{MKO}}$ & 6 & 60 & 1.17 & $51.3 \pm 0.5$ & $0.212 \pm 0.011$ & $138.08 \pm 0.23$ & $238.95 \pm 0.07$ & $0.008 \pm 0.007$ \\
\hline & & & & $J_{\mathrm{MKO}}$ & 6 & 120 & 1.17 & $62 \pm 9$ & $0.062 \pm 0.010$ & $137.54 \pm 0.27$ & $238.72 \pm 0.17$ & $-0.097 \pm 0.004$ \\
\hline & & & & $Y_{\mathrm{NIRC} 2}$ & 5 & 120 & 1.18 & $70 \pm 27$ & $0.036 \pm 0.008$ & $136.7 \pm 3.5$ & $238.4 \pm 0.7$ & $-0.094 \pm 0.063$ \\
\hline WISEA & $\mathrm{J} 114724.10-204021.3$ & 2016 & May 03 & $K_{\mathrm{MKO}}$ & 4 & 60 & 1.33 & $108 \pm 11$ & $0.088 \pm 0.025$ & $\ldots$ & $\ldots$ & $\ldots$ \\
\hline
\end{tabular}

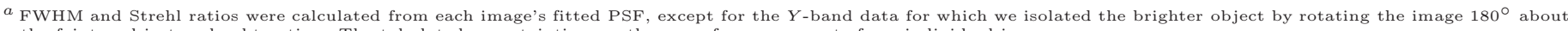
the fainter object and subtracting. The tabulated uncertainties are the rms of measurements from individual images.

The NIRC2 $J, H$, and $K$ filters we used are from the Mauna Kea Observatories (MKO) photometric system (Simons \& Tokunaga 2002; Tokunaga et al. 2002), and the NIRC2 $Y$-band filter is described in Liu et al. (2012). The unresolved photometry reported in K16 is from the VISTA Hemisphere Survey (VHS; PI McMahon, Cambridge, UK), which uses MKO $J$ and $H$ filters but a non-MKO $K_{\mathrm{S}}$ filter. We used the IRTF/SpeX spectrum from K15 to calculate a synthetic $K_{\mathrm{MKO}}=14.658 \pm 0.066 \mathrm{mag}$ for 2MASS J1119-1137, flux-calibrated with K16's $K_{\mathrm{S}}$ magnitude. The VISTA and NIRC2 $Y$ filters are similar enough that no conversion was necessary.

We split the unresolved 2MASS J1119-1137 YJH photometry from K16 and our synthetic $K$ magnitude into resolved photometry using our measured flux ratios (Table 2). $K$-band flux decreases monotonically with spectral type (e.g., Dupuy \& Liu 2012), and the northeast component of 2MASS J1119-1137 is slightly brighter in $K$, so we designate this object as the "A" component. We note a $0.098 \pm 0.014 \mathrm{mag}$ difference between the $K$-band flux ratios measured at the two 


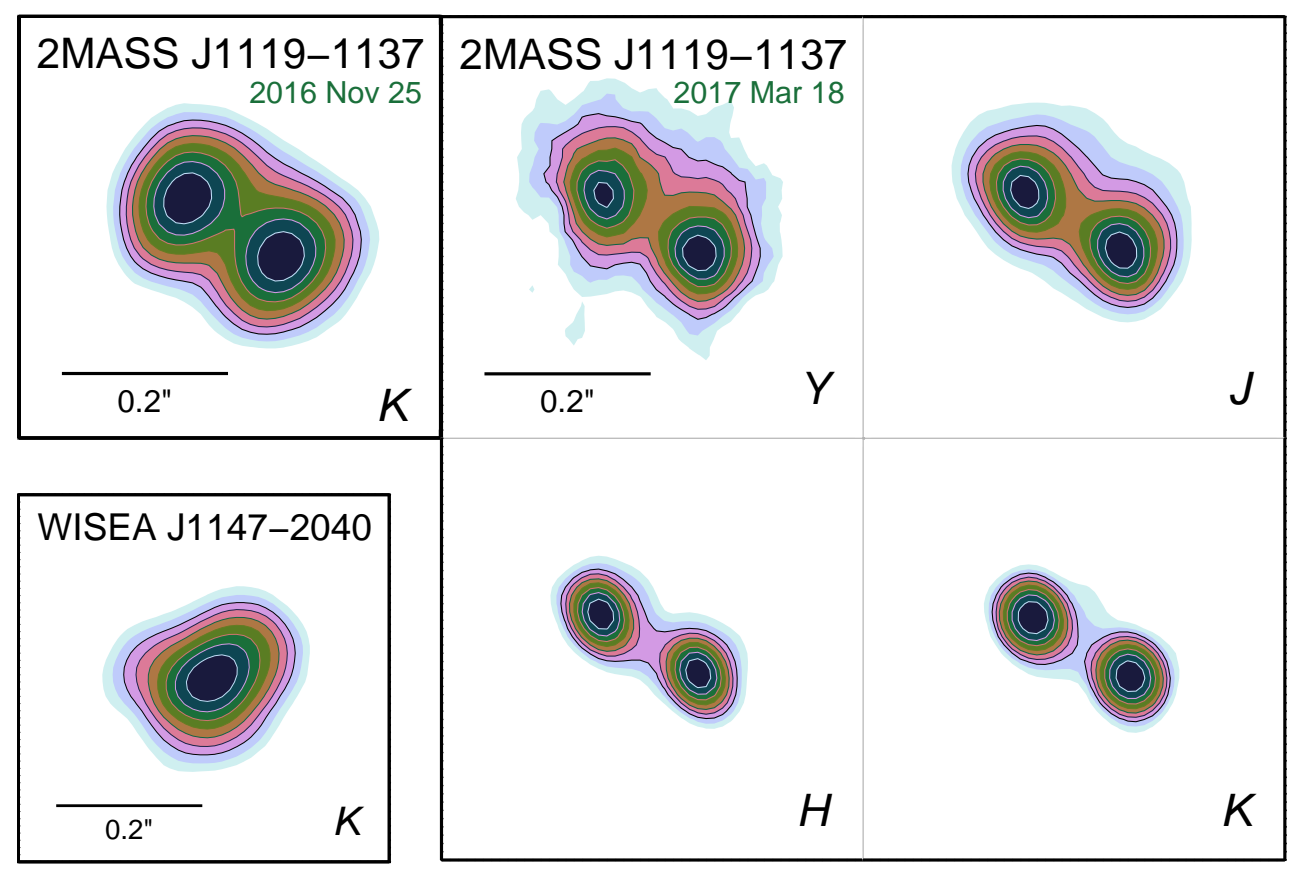

Figure 1. Our Keck LGS AO images of 2MASS J1119-1137 (top left and 2×2 grid at right) and WISEA J1147-2040 (bottom left). North is up and East to the left, with filters indicated at lower right and contours marking logarithmic intervals from $100 \%$ to $10 \%$ of the peak. The northeast component (2MASS J1119-1137A) is marginally brighter in $K$ while the southwest component is brighter in $Y$ and $J$, making this system a possible flux-reversal binary.

epochs. This may indicate variability in one or both components, or systematic errors unaccounted in our uncertainties. We use the 2017 March $K$-band flux ratio for our analysis, as photometry in all other bands was measured that night, and because of the better image quality.

We also identified a faint source in our $J H K$ images from March 2017, lying $3.79 \pm 0.02^{\prime \prime}$ from 2MASS J1119-1137A at PA $=76.47 \pm 0.13 \mathrm{deg}$. It was not well detected but appears to be a point source. Images from the DSS, 2MASS, SDSS, AllWISE, and PanSTARRS1 surveys indicate no object at this location. We measure flux ratios of $4.8 \mathrm{mag}$ in $J, 5.0 \mathrm{mag}$ in $H$, and 5.7 mag in $K$, relative to 2MASS J1119-1137A. At the same distance as 2MASS J1119-1137, this source's $J=22.9 \mathrm{mag}$ would be consistent with known Y0 dwarfs, but its $(J-K)_{\mathrm{MKO}}=1.8 \mathrm{mag}$ color is $\gtrsim 3 \mathrm{mag}$ too red (Leggett et al. 2017). It is almost certainly a background object. Its $(J-K)_{\mathrm{MKO}}$ color suggests an L dwarf, evolved star, or galaxy.

\section{RESULTS}

\subsection{MASS J1119-1137AB is comoving}

Our JHK-band astrometry from March 2017 has a mean separation of $137.88 \pm 0.34$ mas and PA $238.91 \pm$ $0.20 \mathrm{deg}$, with uncertainties estimated as in Section 2. The change in separation from November 2016 is $0.7 \pm 1.5$ mas, consistent with no change. Using the proper motion and photometric distance of 2MASS J1119-1137 (Section 3.3), if 2MASS J1119-1137B were a stationary background object the separation would have decreased by $91.7 \pm$ 9.8 mas in March 2017, inconsistent by $9.4 \sigma$ from our observation (Figure 2). In addition, images from DSS, 2MASS, SDSS, and Pan-STARRS1 showed no objects that could appear as a false close companion given the proper motion. We therefore conclude that 2MASS J1119-1137AB is a gravitationally bound binary.

\subsection{Spectral type and gravity classification}

We used the IRTF/SpeX prism spectrum for 2MASS J1119-1137 and the method of Allers \& Liu (2013) to determine a spectral type of L7, concurring with previous work (K15, Faherty et al. 2016; Gagné et al. 2017). A higher-resolution $J$-band spectrum (K16) shows weakened K I absorption lines having equivalent widths consistent with VL-G classification for L7 dwarfs (Allers \& Liu 2013; Gagné et al. 2017). We therefore adopt L7 VL-G as the unresolved spectral type. The extremely red $(J-K)_{\text {MKO }}$ colors of 2MASS J1119-1137AB are consistent with other lowgravity late-L dwarfs (Figure 3$)$. Both the $(J-K)_{\mathrm{MKO}}$ colors and $K_{\mathrm{MKO}}$ magnitudes for the two components are similar, and the integrated-light spectrum shows no peculiarities that would suggest a blend of two objects with different spectral types. We conclude that both 
Best, W. M. J. ET AL

Table 2. Properties of 2MASS J1119-1137AB

\begin{tabular}{|c|c|c|c|}
\hline Property & $\begin{array}{c}\text { A component } \\
\text { (northeast) }\end{array}$ & $\begin{array}{c}\text { B component } \\
\text { (southwest) }\end{array}$ & Ref. \\
\hline \multicolumn{4}{|c|}{ Observed } \\
\hline R.A. ${ }^{\mathrm{a}}(\mathrm{deg})$ & \multicolumn{2}{|c|}{169.88521} & 1 \\
\hline $\operatorname{Decl}^{\mathrm{a}}(\mathrm{deg})$ & \multicolumn{2}{|c|}{-11.62990} & 1 \\
\hline$\mu_{\alpha} \cos \delta\left(\operatorname{mas~yr}^{-1}\right)$ & \multicolumn{2}{|c|}{$-154.0 \pm 4.0$} & 1 \\
\hline$\mu_{\delta}\left({\left.\operatorname{mas~} \mathrm{yr}^{-1}\right)}^{-1}\right.$ & \multicolumn{2}{|c|}{$-107.9 \pm 1.8$} & 1 \\
\hline Radial velocity $\left(\mathrm{km} \mathrm{s}^{-1}\right)$ & \multicolumn{2}{|c|}{$8.5 \pm 3.3$} & 2 \\
\hline $\mathrm{SpT}$ & \multicolumn{2}{|c|}{ L7 VL-G } & $3,4,5$ \\
\hline$Y$ (mag) & \multicolumn{2}{|c|}{$19.045 \pm 0.093$} & 2 \\
\hline$J$ (mag) & \multicolumn{2}{|c|}{$17.330 \pm 0.029$} & 2 \\
\hline$H(\mathrm{mag})$ & \multicolumn{2}{|c|}{$15.884 \pm 0.017$} & 2 \\
\hline$K$ (mag) & \multicolumn{2}{|c|}{$14.658 \pm 0.066^{\mathrm{b}}$} & 3 \\
\hline$Y(\mathrm{mag})$ & $19.84 \pm 0.10$ & $19.75 \pm 0.10$ & 3 \\
\hline$J$ (mag) & $18.13 \pm 0.03$ & $18.04 \pm 0.03$ & 3 \\
\hline$H$ (mag) & $16.59 \pm 0.02$ & $16.60 \pm 0.02$ & 3 \\
\hline$K(\mathrm{mag})$ & $15.40 \pm 0.07$ & $15.43 \pm 0.07$ & 3 \\
\hline$Y-J(\mathrm{mag})$ & $1.71 \pm 0.10$ & $1.71 \pm 0.10$ & 3 \\
\hline$J-H(\mathrm{mag})$ & $1.54 \pm 0.03$ & $1.43 \pm 0.03$ & 3 \\
\hline$J-K(\operatorname{mag})$ & $2.73 \pm 0.07$ & $2.61 \pm 0.07$ & 3 \\
\hline \multirow[t]{2}{*}{$\Delta(J-K)(\mathrm{mag})$} & \multicolumn{2}{|c|}{$0.125 \pm 0.011$} & 3 \\
\hline & \multicolumn{3}{|l|}{ Estimated } \\
\hline$d_{\text {phot }}(\mathrm{pc})$ & \multicolumn{2}{|c|}{$26.4 \pm 6.9$} & 3,8 \\
\hline$m-M(\mathrm{mag})$ & \multicolumn{2}{|c|}{$2.11 \pm 0.56$} & 3 \\
\hline$v_{\tan }\left(\mathrm{km} \mathrm{s}^{-1}\right)$ & \multicolumn{2}{|c|}{$23.6 \pm 6.1$} & 3 \\
\hline Projected separation (AU) & \multicolumn{2}{|c|}{$3.6 \pm 0.9$} & 3 \\
\hline Semi-major axis $(A U)$ & \multicolumn{2}{|c|}{$3.9_{-1.4}^{+1.9}$} & 3 \\
\hline Orbital Period (yr) & \multicolumn{2}{|c|}{$90_{-50}^{+80}$} & 3 \\
\hline $\log \left(L_{\mathrm{bol}} / L_{\odot}\right)($ unresolved $)$ & \multicolumn{2}{|c|}{$-4.44_{-0.27}^{+0.21}$} & 3,6 \\
\hline $\log \left(L_{\mathrm{bol}} / L_{\odot}\right)($ resolved $)$ & $-4.73_{-0.27}^{+0.21}$ & $-4.74_{-0.27}^{+0.21}$ & 3 \\
\hline
\end{tabular}

Model-derived (Lyon/DUSTY) assuming TWA membership

\begin{tabular}{|c|c|c|c|}
\hline Age (Myr) & \multicolumn{2}{|c|}{$10 \pm 3 \mathrm{Myr}$} & 7 \\
\hline $\operatorname{Mass}\left(M_{\mathrm{Jup}}\right)$ & $3.7_{-0.9}^{+1.2}$ & $3.7_{-0.9}^{+1.2}$ & 3 \\
\hline$T_{\text {eff }}(\mathrm{K})$ & $1013_{-109}^{+122}$ & $1006_{-109}^{+122}$ & 3 \\
\hline
\end{tabular}

\begin{tabular}{lccc}
\multicolumn{4}{c}{ Model-derived (Lyon/DUSTY) assuming young field (VL-G) } \\
\hline Age $(\mathrm{Myr})$ & $10-100 \mathrm{Myr}$ & 8 \\
Mass $\left(M_{\mathrm{Jup}}\right)$ & $9.2_{-1.9}^{+2.3}$ & $9.0_{-1.9}^{+2.4}$ & 3 \\
$T_{\text {eff }}(\mathrm{K})$ & $1065_{-118}^{+133}$ & $1059_{-118}^{+133}$ & 3 \\
\hline
\end{tabular}

NOTE- $-J H K$ photometry is on the MKO system. $Y$ photometry is from similar filters on VISTA (integrated light) and Keck/NIRC2 (resolved); no conversion was performed.

$a_{\text {Epoch }} 54858.45$ (MJD).

${ }^{b}$ Synthetic photometry based on the SpeX prism spectrum (K15) and VHS $K_{\mathrm{S}}$ photometry (K16).

References-(1) Best et al. (2017), (2) K16, (3) this work, (4) K15, (5) Gagné et al. (2017), (6) Faherty et al. (2016), (7) Bell et al. (2015), (8) Liu et al. (2016). 


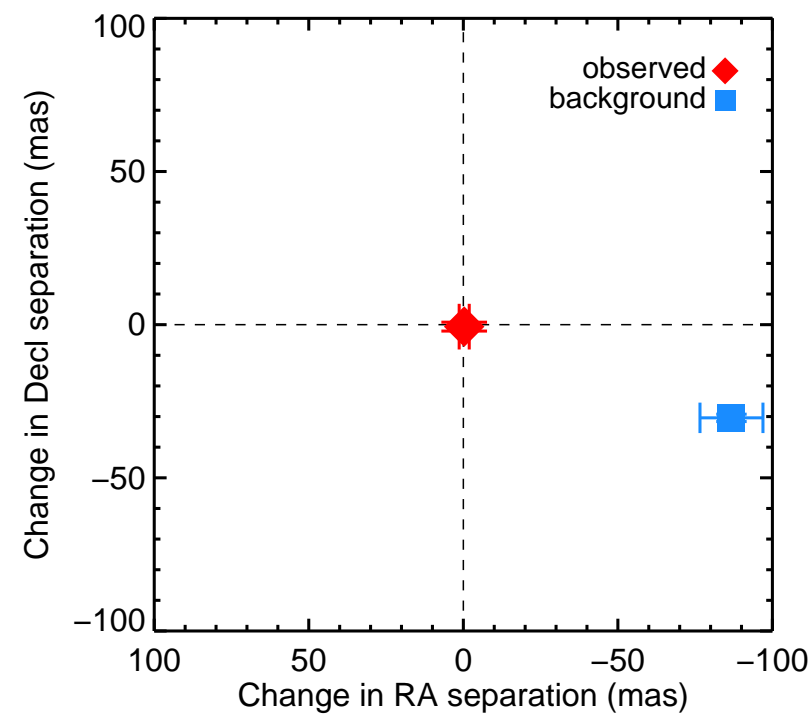

Figure 2. The change in position of 2MASS J1119-1137B with respect to 2MASS J1119-1137A between 2016 November 25 and 2017 March 18 (red diamond), compared with the change expected due to parallax and proper motion of 2MASS J1119-1137A if 2MASS J1119-1137B were a stationary background object (blue square). The error for the background position is dominated by the uncertainty on our photometric distance. The observed positions of 2MASS J1119-1137B are consistent with no change, while the background position differs by $9.4 \sigma$, confirming that 2MASS J1119-1137AB is a binary.

2MASS J1119-1137A and 2MASS J1119-1137B have spectral types L7 VL-G.

\subsection{Distance}

We used the spectral-type-to- $M_{K_{\mathrm{MKO}}}$ relation for VL-G dwarfs from Liu et al. (2016) and our resolved photometry to estimate photometric distances of $26.3 \pm$ $6.8 \mathrm{pc}$ and $26.6 \pm 6.9 \mathrm{pc}$ for 2MASS J1119-1137A and 2MASS J1119-1137B, respectively. We adopt $26.4 \pm$ $6.9 \mathrm{pc}$ as the distance to the system, giving the binary a projected separation of $3.6 \pm 0.9 \mathrm{AU}$. Our distance is consistent with K16's kinematic distance of $28.9 \pm 3.6 \mathrm{pc}$ assuming membership in TWA.

\subsection{TWA Membership}

K16 identified 2MASS J1119-1137 as a candidate TWA member, using the BANYAN II online tool (Malo et al. 2013; Gagné et al. 2014) to calculate an $88 \%$ membership probability with a contamination probability of $0.003 \%$ based on 2MASS J1119-1137's sky position, proper motion, radial velocity, and youth. Faherty et al. (2016) analyzed moving group membership using four different tools and found $>90 \%$ probabilities for TWA in three cases; LACEwING (Riedel et al. 2017) found a contrasting probability of
16\%. Gagné et al. (2017) used the full BANYAN II analysis (including photometry) to calculate a $97 \%$ probability of membership. Liu et al. (2016) found that objects lacking parallaxes which have full BANYAN II probabilities $\gtrsim 80 \%$ tend to have memberships confirmed by subsequent parallax measurements for the well-established moving groups, including TWA.

We reassessed the moving group membership of 2MASS J1119-1137 using the BANYAN II online tool. For position and proper motion, we adopted the values from Best et al. (2017), which are calculated from

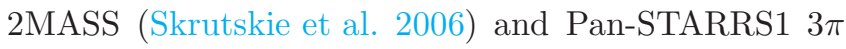
(Chambers et al. 2017; Magnier et al. 2017) astrometry and calibrated to the Gaia DR1 reference frame (Gaia Collaboration et al. 2016; Lindegren et al. 2016).

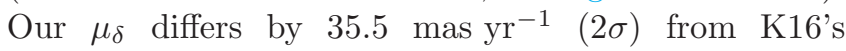
value but is consistent with measurements by K15 and Gagné et al. (2017).

Using only our astrometry and the radial velocity from K16 as inputs to BANYAN II, we obtained a TWA membership probability of $73 \%$. The decrease from K16's $88 \%$ probability is due almost entirely to the difference in $\mu_{\delta}$. When we included our photometric distance, the probability of TWA membership increased to $82 \%$.

Based on our BANYAN II results and the VL-G spectrum, 2MASS J1119-1137 is a very likely member of TWA. We note that a comparison in UVW and XYZ of 2MASS J1119-1137 to known members of moving groups using $\tilde{\chi}^{2}$ as a rubric (see Equation 1 of Liu et al. 2016) supports membership in TWA, $\beta$ Pictoris $(0 \%$ probability from BANYAN II), and Tucana-Horologium (0\%), and especially the $\mathrm{AB}$ Doradus (4\%) moving group. A precise trigonometric distance, now underway by us at CFHT, is needed to firmly establish the membership.

\subsection{Physical Properties}

As a TWA member, 2MASS J1119-1137 would share the age of $10 \pm 3 \mathrm{Myr}$ derived from the stellar members (Bell et al. 2015). We estimated the components' masses and effective temperatures using bolometric luminosities and the Lyon/DUSTY hot-start evolutionary models (Chabrier et al. 2000). To obtain $L_{\text {bol }}$, we adjusted the integrated-light value from Faherty et al. (2016) to our new photometric distance, and decomposed this into individual $L_{\mathrm{bol}}$ values using the binary's $K_{\mathrm{MKO}}$ flux ratio. Using these $L_{\mathrm{bol}}$ and the assumed age, we then interpolated a mass and effective temperature from the DUSTY models for each component. We propagated the uncertainties on distance, flux ratio, and age into our calculations via Monte Carlo trials using normal distributions for each uncertainty, and we quote the resulting median and $68 \%$ confidence limits (Table 2). We estimate masses of $3.7_{-0.9}^{+1.2} M_{\text {Jup }}$ for both compo- 


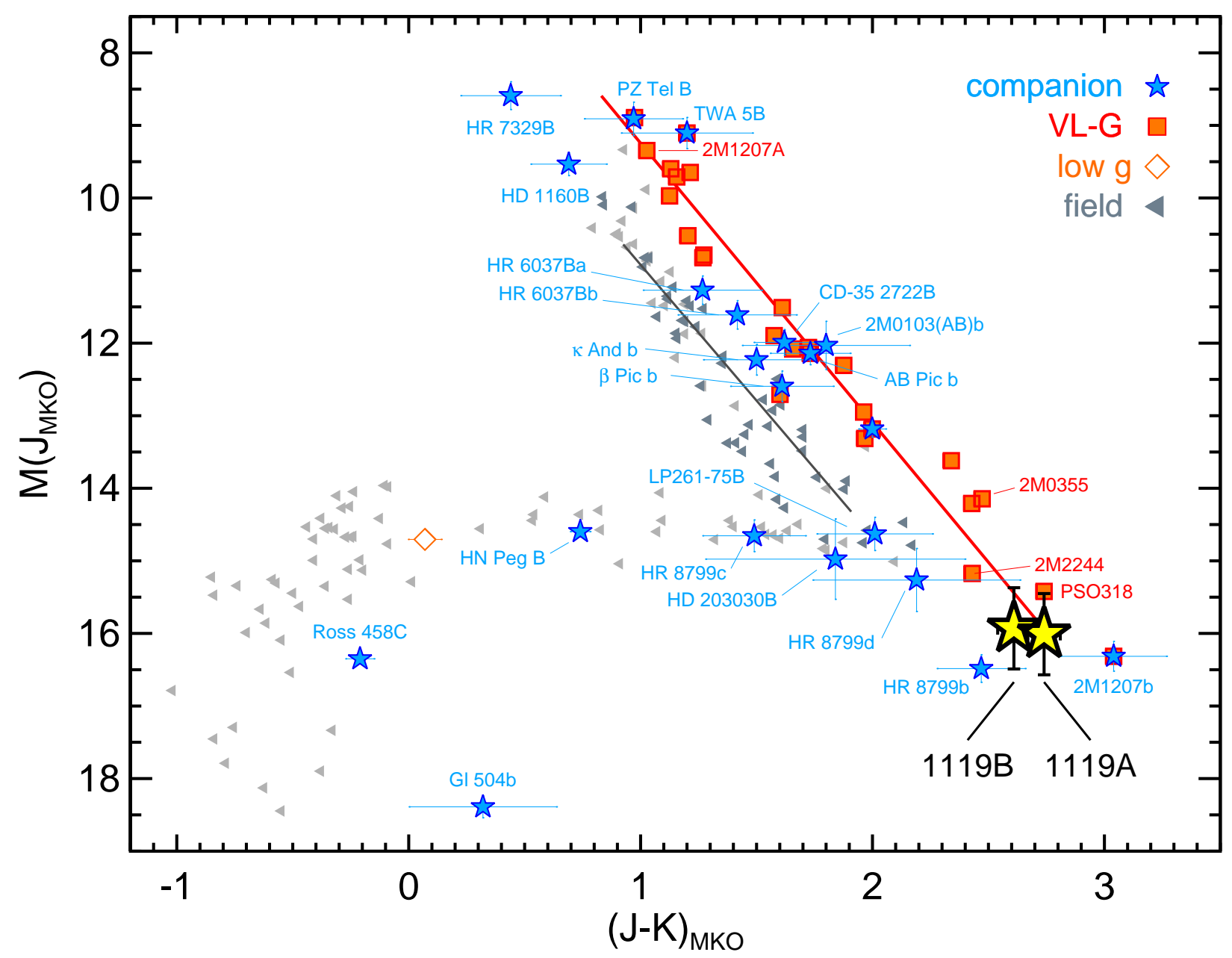

Figure 3. $J_{\mathrm{MKO}}$ vs. $(J-K)_{\mathrm{MKO}}$ color-magnitude diagram for ultracool dwarfs having parallaxes (adapted from Liu et al. 2016). Gray triangles indicate field brown dwarfs, red squares indicate late-M and L dwarfs with VL-G classifications, and blue stars indicate companions. Gray and red lines show linear fits for the FLD-G and VL-G dwarfs, respectively. 2MASS J1119-1137AB (yellow stars, using our photometric distance) lies among the faintest and reddest planetary-mass L dwarfs. The $M_{J_{\mathrm{MKO}}}$ errors for 2MASS J1119-1137AB are dominated by the $d_{\text {phot }}$; we measure $\Delta J_{\mathrm{MKO}}=-0.097 \pm 0.004$ mag. The relative positions of 2MASS J1119-1137AB imply that 2MASS J1119-1137B has begun the transition from a red L dwarf to a bluer T dwarf.

nents. We did not use the more recent BHAC15 models (Baraffe et al. 2015) as they do not include masses below $0.01 M_{\odot}$.

If 2MASS J1119-1137 is not a TWA member, its VL-G classification still constrains its age to $\approx 10-$ 100 Myr (Liu et al. 2016). Our estimated masses and effective temperatures for this age range (uniformly distributed for error propagation) are shown in Table 2.

Using our masses, we considered the effect of 2MASS J1119-1137AB's orbital motion on the radial velocity measured by K16. For an edge-on circular orbit with components at quadrature, we found a difference in radial velocities of $1.3_{-0.3}^{+0.4} \mathrm{~km} \mathrm{~s}^{-1}$ for TWA masses or $2.0_{-0.4}^{+0.6} \mathrm{~km} \mathrm{~s}^{-1}$ for field-VL-G masses. The $R \sim 6000$ $\left(50 \mathrm{~km} \mathrm{~s}^{-1}\right)$ spectrum of K16 would not resolve such orbital motion, so the radial velocity from K16 remains valid.

\section{DISCUSSION}

Assuming 2MASS J1119-1137 is a member of TWA, its total mass is $7.4_{-1.9}^{+2.5} M_{\text {Jup }}$, making it the lowestmass binary discovered to date. The individual masses of 2MASS J1119-1137A and 2MASS J1119-1137B also place them among the lowest-mass free-floating brown dwarfs, including WISEA J1147-2040 and the Y dwarfs (Dupuy \& Kraus 2013; Leggett et al. 2017). Even if 2MASS J1119-1137AB is actually a young field object, its total mass of $18.2_{-3.8}^{+4.7} M_{\text {Jup }}$ would still be among the lowest-mass binaries, surpassed only by the young binary DENIS-P J035726.9441730 (14-15 Mup; Bouy et al. 2003; Gagné et al. 2014) and possibly by the T9+Y0 binaries CFBDSIR J145829+101343 ( $\approx 18-45 \quad M_{\text {Jup }}$; Liu et al. 2011) and WISE J014656.66+423410.0 (Dupuy et al. 
2015a). As an extremely low-mass young binary, 2MASS J1119-1137AB will be a crucial benchmark for tests of evolutionary and atmospheric models.

The isolation of 2MASS J1119-1137AB strongly suggests that it is a product of normal star-formation processes, which therefore must be capable of making binaries with $\lesssim 5 M_{\text {Jup }}$ components. 2MASS J1119-1137AB could be a fragment of a higher-order system that was ejected via dynamical interactions (Reipurth \& Mikkola 2015), although the lack of any confirmed member of TWA within $10^{\circ}$ (projected separation $\approx$ 5 pc) of 2MASS J1119-1137 makes this scenario unlikely. Formation of very low mass binaries in extended massive disks around Sun-like stars followed by ejection into the field has been proposed by, e.g., Stamatellos \& Whitworth (2009), but disks of this type have not been observed.

Binary brown dwarfs can be monitored to map their orbits, which yield dynamical masses that stringently test evolutionary models. We estimated 2MASS J1119-1137AB's orbital period using Kepler's Third Law. We first used the projected separation and a conversion factor from Dupuy \& Liu (2011, Table 6, assuming moderate discovery bias for very low-mass visual binaries) to estimate a semi-major axis of $3.9_{-1.4}^{+1.9} \mathrm{AU}$. Our model-derived masses assuming TWA membership give an orbital period of $90_{-50}^{+80} \mathrm{yr}$. A dynamical mass can be determined once $\approx 1 / 3$ of an orbit has been observed (e.g., Dupuy \& Liu 2017), so 2MASS J1119-1137AB would yield a dynamical mass in $\approx 15-55$ years. Assuming a field VL-G age, we estimate an orbital period of $60_{-30}^{+50} \mathrm{yr}$, yielding a mass in $\approx 10-35$ years.

The integrated-light spectrum and photometry of 2MASS J1119-1137 are notably similar to those of the young L7 dwarf WISEA J1147-2040 (Gagné et al. 2017), implying similar temperatures and gravity. However, using the $L_{\text {bol }}$ for WISEA J1147-2040 from Faherty et al. (2016) and the method from Section 3.5, we estimate a $T_{\text {eff }}$ of $1242_{-69}^{+73} \mathrm{~K}$, which is $\approx 230 \mathrm{~K}$ higher than our estimates for 2MASS J1119-1137A and 2MASS J1119-1137B. This discrepancy is particularly surprising given that both objects are very likely members of TWA and therefore should have the same age and composition. The simplest resolution is that WISEA J1147-2040 is also an equal-flux binary, unresolved in our images, with component temperatures very similar to those of 2MASS J1119-1137AB. Using the method from Section 3.3 we calculated $d_{\text {phot }}=27.3 \pm 6.9 \mathrm{pc}$ for a hypothetical equalflux binary WISEA J1147-2040, leading to a maximum projected separation of $2.9 \pm 0.8 \mathrm{AU}$ to remain unresolved in our images. Another possibility is that 2MASS J1119-1137AB is not a member of TWA and is older; our VL-G-age temperature estimates are only $\approx 50 \mathrm{~K}$ higher, but would then agree with the WISEA J1147-2040 estimate within uncertainties. A third intriguing possibility is that the low-resolution spectra of young red L dwarfs are driven at least partially by factors other than temperature and gravity (Allers \& Liu 2013; Liu et al. 2016), allowing coeval objects with differing masses and effective temperatures to have similar spectra.

Figure 3 compares the $J_{\mathrm{MKO}}$ vs. $(J-K)_{\mathrm{MKO}}$ position of 2MASS J1119-1137AB to the ultracool dwarf population, highlighting other low-gravity objects and substellar companions. 2MASS J1119-1137AB lies among other planetary-mass objects at the faint red end of the L dwarf sequence. 2MASS J1119-1137B is brighter in $J$ band and slightly fainter in $K$ band, making the system a probable flux-reversal binary. (The $K$ magnitudes for the two components are formally consistent within uncertainties, but 2MASS J1119-1137B is fainter in all nine of our individual $K$-band images.) The flux-reversal phenomenon is a hallmark of field-age L/T transition binaries (e.g., Gizis et al. 2003; Liu et al. 2006; Dupuy et al. 2015b), thought to occur when the cooler component reaches a temperature at which the clouds that suppress near-IR flux in L dwarfs begin to clear, reducing the $J$-band opacity relative to the warmer component (e.g., Burrows et al. 2006). The slightly bluer $(J-K)_{\mathrm{MKO}}$ color of $2 \mathrm{MASS}$ J1119-1137B implies that it is cooler than its primary. In fieldage brown dwarfs this transition to bluer $J-K$ colors is typically seen at warmer temperatures $(\approx 1400 \mathrm{~K}$; e.g., Dupuy \& Liu 2012). The potential flux-reversal of 2MASS J1119-1137AB suggests that it is beginning the transition at $T_{\text {eff }} \approx 1000 \pm 100 \mathrm{~K}$, an even lower temperature than the $\approx 1100-1200 \mathrm{~K}$ found for other low (but somewhat higher) mass L dwarfs (e.g. Metchev \& Hillenbrand 2006; Barman et al. 2011; Liu et al. 2013), implying a possible systematic correlation between mass and $\mathrm{L} / \mathrm{T}$ transition temperature. Precise photometry and resolved spectroscopy of 2MASS J1119-1137AB with the Hubble and James Webb Space Telescopes will enable differential studies of the atmospheres of young planetary-mass objects and may yield insights into the $\mathrm{L} / \mathrm{T}$ transition at young ages.

We thank the referee for an immediate and helpful review. The IRTF/SpeX spectrum for 2MASS J1119-1137 was retrieved from the SpeX Prism Library, maintained by Adam Burgasser at http://www.browndwarfs.org/spexprism. The PanSTARRS1 Surveys have been made possible through contributions of the Institute for Astronomy, the University of Hawaii, the Pan-STARRS Project Office, the Max-Planck Society and its participating institutes, the 
Max Planck Institute for Astronomy, Heidelberg and the Max Planck Institute for Extraterrestrial Physics, Garching, The Johns Hopkins University, Durham University, the University of Edinburgh, Queen's University Belfast, the Harvard-Smithsonian Center for Astrophysics, the Las Cumbres Observatory Global Telescope Network Incorporated, the National Central University of Taiwan, the Space Telescope Science Institute, the National Aeronautics and Space Administration under Grant No. NNX08AR22G issued through the Planetary Science Division of the NASA Science Mission Directorate, the National Science Foundation under Grant No. AST-1238877, the University of Maryland, Eotvos Lorand University (ELTE), and the Los Alamos
National Laboratory. This work has made use of data from the European Space Agency (ESA) mission Gaia (http://www. cosmos.esa.int/gaia), processed by the Gaia Data Processing and Analysis Consortium (DPAC, http://www. cosmos.esa.int/web/gaia/dpac/consortium). WMJB and MCL received support from NSF grant AST-1518339. WMBJ, MCL, and EAM received support from NSF grant AST-1313455. Finally, the authors acknowledge the significance of the summit of Maunakea for the Native Hawaiian community. We are fortunate to have the opportunity to conduct observations from this mountain.

Facility: Keck, Pan-STARRS1, Gaia

\section{REFERENCES}

Allers, K. N., \& Liu, M. C. 2013, ApJ, 772, 79

Baraffe, I., Homeier, D., Allard, F., \& Chabrier, G. 2015, A\&A, 577, A42

Barman, T. S., Macintosh, B., Konopacky, Q. M., \& Marois, C. 2011, ApJ, 733, 65

Bell, C. P. M., Mamajek, E. E., \& Naylor, T. 2015, MNRAS, 454, 593

Best, W. M. J., Magnier, E. A., Liu, M. C., et al. 2017, AAS Journals, submitted, arXiv:1701.00490

Bouy, H., Brandner, W., Martín, E. L., et al. 2003, AJ, 126, 1526

Burrows, A. S., Sudarsky, D., \& Hubeny, I. 2006, ApJ, 640, 1063

Chabrier, G., Baraffe, I., Allard, F., \& Hauschildt, P. 2000, ApJ, 542,464

Chambers, K. C., Magnier, E. A., Metcalfe, N., et al. 2017, in preparation, arXiv: 1612.05560

Cruz, K. L., Kirkpatrick, J. D., \& Burgasser, A. J. 2009, AJ, 137,3345

Diolaiti, E., Bendinelli, O., Bonaccini, D., et al. 2000, A\&AS, 147,335

Dupuy, T. J., \& Kraus, A. L. 2013, Science, 341, 1492

Dupuy, T. J., \& Liu, M. C. 2011, ApJ, 733, 122

-. 2012, ApJS, 201, 19

—. 2017, ApJS, in press, arXiv:1703.05775

Dupuy, T. J., Liu, M. C., \& Ireland, M. J. 2009, ApJ, 692, 729

Dupuy, T. J., Liu, M. C., \& Leggett, S. K. 2015a, ApJ, 803, 102

Dupuy, T. J., Liu, M. C., Leggett, S. K., et al. 2015b, ApJ, 805, 56

Faherty, J. K., Riedel, A. R., Cruz, K. L., et al. 2016, ApJS, 225, 10

Gagné, J., Lafreniere, D., Doyon, R., Malo, L., \& Artigau, E. 2014, ApJ, 783, 121

Gagné, J., Faherty, J. K., Mamajek, E. E., et al. 2017, ApJS, 228, 18

Gaia Collaboration, Prusti, T., de Bruijne, J. H. J., et al. 2016, A\&A, 595, A1

Gizis, J. E., Reid, I. N., Knapp, G. R., et al. 2003, AJ, 125, 3302

Kellogg, K., Metchev, S. A., Gagné, J., \& Faherty, J. 2016, ApJL, 821, L15

Kellogg, K., Metchev, S. A., Geißler, K., et al. 2015, AJ, 150, 182
Leggett, S. K., Tremblin, P., Esplin, T. L., Luhman, K. L., \& Morley, C. V. 2017, ApJ, in press, arXiv:1704.03573

Lindegren, L., Lammers, U., Bastian, U., et al. 2016, A\&A, 595, A4

Liu, M. C., Dupuy, T. J., \& Allers, K. N. 2016, ApJ, 833, 96

Liu, M. C., Dupuy, T. J., Bowler, B. P., Leggett, S. K., \& Best, W. M. J. 2012, ApJ, 758, 57

Liu, M. C., Dupuy, T. J., \& Ireland, M. J. 2008, ApJ, 689, 436

Liu, M. C., Leggett, S. K., Golimowski, D. A., et al. 2006, ApJ, 647,1393

Liu, M. C., Delorme, P., Dupuy, T. J., et al. 2011, ApJ, 740, 108

Liu, M. C., Magnier, E. A., Deacon, N. R., et al. 2013, ApJL, 777, L20

Magnier, E. A., Schlafly, E. F., Finkbeiner, D. P., et al. 2017, in preparation, arXiv: 1612.05242

Malo, L., Doyon, R., Lafreniere, D., et al. 2013, ApJ, 762, 88

Metchev, S. A., \& Hillenbrand, L. A. 2006, ApJ, 651, 1166

Monet, D. G., Levine, S. E., Canzian, B., et al. 2003, AJ, 125, 984

Reipurth, B., \& Mikkola, S. 2015, AJ, 149, 145

Riedel, A. R., Blunt, S. C., Lambrides, E. L., et al. 2017, AJ, 153, 95

Schneider, A. C., Windsor, J., Cushing, M. C., Kirkpatrick, J. D., \& Wright, E. L. 2016, ApJL, 822, L1

Service, M., Lu, J. R., Campbell, R., et al. 2016, PASP, 128, 095004

Simons, D. A., \& Tokunaga, A. T. 2002, PASP, 114, 169

Skrutskie, M. F., Cutri, R. M., Stiening, R., et al. 2006, AJ, 131, 1163

Stamatellos, D., \& Whitworth, A. P. 2009, MNRAS, 392, 413

Tokunaga, A. T., Simons, D. A., \& Vacca, W. D. 2002, PASP, 114,180

van Dam, M. A., Bouchez, A. H., Le Mignant, D., et al. 2006, PASP, 118, 310

Webb, R. A., Zuckerman, B., Platais, I., et al. 1999, ApJ, 512, L63

Wizinowich, P. L., Le Mignant, D., Bouchez, A. H., et al. 2006, PASP, 118, 297 